\title{
Hystoroscopy Assisted Non-Suture Cold-Knife Conization and Cold-Knife Conization in Patients with CINIII: A Comparative Study
}

\author{
XiongJ, Deng Y and Zhang H* \\ Department of Gynecology, The Second Xiangya Hospital, \\ Central South University, Changsha, China \\ *Corresponding author: Hongwen Zhang, \\ Department of Gynecology, The Second Xiangya Hospital, \\ Central South University, Changsha, China
}

Received: April 27, 2021; Accepted: May 24, 2021;

Published: May 31, 2021

\begin{abstract}
167 patients of cervical conization were performed for CINIII in a 34-month study. Conization include Cold-Knife Conization (CKC) $(n=120)$ and hystoroscopy assisted non-suture cold-knife conization (HCKC) $(n=47)$. Both groups were similar in terms of age, indications for conization, and size of cervical cone specimens. Comparing with CKC group, HCKC group was done in a significantly shorter time (28.52 \pm 9.80 minutes vs. $52.19 \pm 18.09$ minutes) $(P<0.05)$, with less intraoperative bleeding of HCKC group $(44.89 \pm 10.81 \mathrm{ml}$ vs. $51.50 \pm 19.94 \mathrm{ml})(\mathrm{P}<0.05)$, shorter hospitalization time $(6.89 \pm 2.11$ days vs. $7.63 \pm 1.40$ days $)(P<0.05$, and higher HPV clearance rate $(100 \%$ vs. $87.13 \%)$ $(P<0.05)$ than CKC group. While, the difference in the cure rate $(95 \%$ vs. $91 \%)$ $(P>0.05)$, the recurrence rate $(0 \%$ vs. $1 \%)(P>0.05)$ and the postoperative complication $(P>0.05)$ between HCKC group and CKC group were not significant. The margins of the excision were checked by rapid histologic examination, showing the incidence of residual CIN was lower in HCKC group $(8.51 \%)$ than in CKC group $(9.17 \%)(P>0.05)$. Our study indicates that HCKC is a better treatment than CKC in the management of patients with CINIII.
\end{abstract}

Keywords: Cold-Knife Conization; Cervical Intraepithelial Neoplasia III; Hystoroscopy

\section{Introduction}

The incidence of Cervical Intraepithelial Neoplasia III (CINIII) is increasing particularly in young women, requiring conservative treatment. Conization is the preferred operation method for patients with CINIII who has fertility requirement.

Conization of cervix refers to remove part of cervix, including the cervical transitional band and part or all of the cervical canal tissue [1]. Cold-Knife Conization (CKC) is the most common option in conization. It can obtain complete specimen tissue and not affect pathological diagnosis [2]. In addition, CKC has high cure rate and low recurrence rate in CINIII [3]. However, limited surgical field and difficulty in suture due to the small vaginal route is the most prominent shortcoming of CKC. In addition, the cervix shape is hard to restore to normal after cold-knife conization.

Recently, Hystoroscopy assisted non-suture Cold-Knife Conization (HCKC) is encouraged, which use roller electrocoagulation under hysteroscope for hemostasis after removing cervical lesions with cold-knife [4]. Few clinical trials comparing the HCKC to the $\mathrm{CKC}$ technique was reported. The aim of this study is to compare HCKC with CKC in terms of the time required to complete excision ,the amount of bleeding in operation, the incidence of residual lesion from subsequent hysterectomy specimen, the hospitalization time, the HPV clearance rate, cure rate, recurrence rate and postoperative complications.

\section{Materials and Methods}

In this study, we analyzed the clinical data of 167 patients with
CINIII who were treated with HCKC and CKC in the Department of Gynecology at the second XiangYa Hospital, Central South University, China, between December 2016 and October 2019. The diagnosis of CINIII had been confirmed by histopathology with cervical cone biopsy.

CKC $(n=120)$ were performed under general anesthesia, lumbar anesthesia, epidural anesthesia, lumbar-epidural-combined anesthesia or caudal anesthesia. Cold knife was used to cut a cone after normal saline uterocervical injection. Then suture cervical wound with absorbable line. HCKC $(n=47)$ were performed under general intravenous anesthesia. Ball diathermy with Hysteroscope was used to coagulate the surface of the wound to control any active bleeding after removing the cone.

The statistical analysis was carried out with SPSS version 23.0 software and compared with independent $t$-test or chi-square test.

\section{Results}

\section{Demographics of the patients}

The demographics of CINIII patients are shown in Figure 1. There was no statistically significant difference of age between the two groups. Both group showed the peak age for CINIII were between 36 and 40 years old.

\section{Analysis of intraoperative and postoperative data}

Intraoperative data are shown in Table 1, including the operative time, intraoperative bleeding and the postoperative CIN residual lesion checked by histological examination. The average operative 


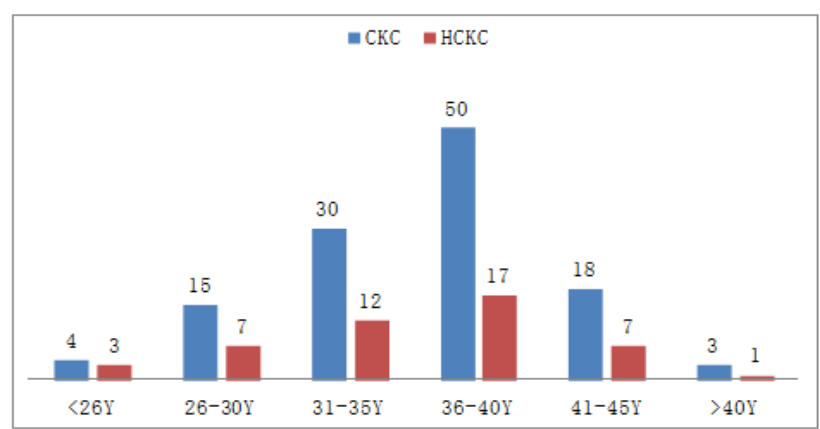

Figure 1: Age distribution of patients with cervical intraepithelial neoplasis.

Table 1: Comparison of the results with HCKC group and CKC group.

\begin{tabular}{|c|c|c|c|}
\hline & HCKC $(\mathbf{n}=\mathbf{4 7})$ & CKC $(\mathbf{n = 1 2 0})$ & P \\
\hline Operative time & $28.52 \pm 9.80$ & $52.19 \pm 18.09$ & $<0.05$ \\
\hline Intraoperative bleeding & $44.89 \pm 10.81$ & $51.50 \pm 19.94$ & $<0.05$ \\
\hline Residual lesion & $4(8.51 \%)$ & $11(9.17 \%)$ & $>0.05$ \\
\hline
\end{tabular}

Table 2: Comparison the hospital stays and the medical costs.

\begin{tabular}{|c|c|c|c|}
\hline & HCKC $(\mathbf{n}=\mathbf{4 7})$ & CKC $(\mathbf{n}=\mathbf{1 2 0})$ & P \\
\hline Hospital stay (days) & $6.89 \pm 2.11$ & $7.63 \pm 1.40$ & $<0.05$ \\
\hline Medical costs (yuan) & $12000.83 \pm 3813.16$ & $13492.11 \pm 3120.11$ & $<0.05$ \\
\hline
\end{tabular}

Table 3: Comparison the cure rate, HPV clearance rate and the recurrence rate.

\begin{tabular}{|c|c|c|c|}
\hline & HCKC $(\mathbf{n}=\mathbf{4 0})$ & CKC $(\mathbf{n = 1 0 1 )}$ & P \\
\hline Cure rate & $38(95 \%)$ & $92(91.09 \%)$ & $>0.05$ \\
\hline HPV negative rate & $40(100 \%)$ & $88(87.12 \%)$ & $<0.05$ \\
\hline Recurrence rate & 0 & $1(0.99 \%)$ & $>0.05$ \\
\hline
\end{tabular}

Table 4: Comparison the postoperative complications.

\begin{tabular}{|c|c|c|c|}
\hline & HCKC & CKC & P \\
\hline Infection & $2(47)$ & $11(120)$ & $>0.05$ \\
\hline Postoperative hemorrhages & $2(47)$ & $2(120)$ & $>0.05$ \\
\hline Cervical adhesion & $0(40)$ & $4(101)$ & $>0.05$ \\
\hline
\end{tabular}

time and intraoperative bleeding in HCKC group were significantly lower than in CKC group $(\mathrm{P}<0.05)$. The CIN residual lesion treated by HCKC was fewer than by $\mathrm{CKC}$, while the difference was not statistically different $(\mathrm{P}>0.05)$. Patients who had residual lesions after the operation did hysterectomy. Two patients in the CKC group who had residual lesion did cervical conization again because of desiring conserving fertility and no residual lesion existed.

Hospital stay and medical expenses in HCKC group were much less than that in CKC group $(\mathrm{p}<0.05)$ (Table 2).

141 patients of 154 completed postoperative follow-up (40 cases in HCKC group and 101 cases in CKC group), except the other 13 patients of 167 who experienced hysterectomy. Table 3 shows the clearance of HPV. All patients in HCKC group turned negative (100\%), which was significantly higher than in CKC group (87.12\%) $(\mathrm{P}<0.05)$. Although the cure rate of HCKC group was higher than that of CKC group, and the recurrence rate in HCKC group was zero in HCKC group, the difference between the two groups were not

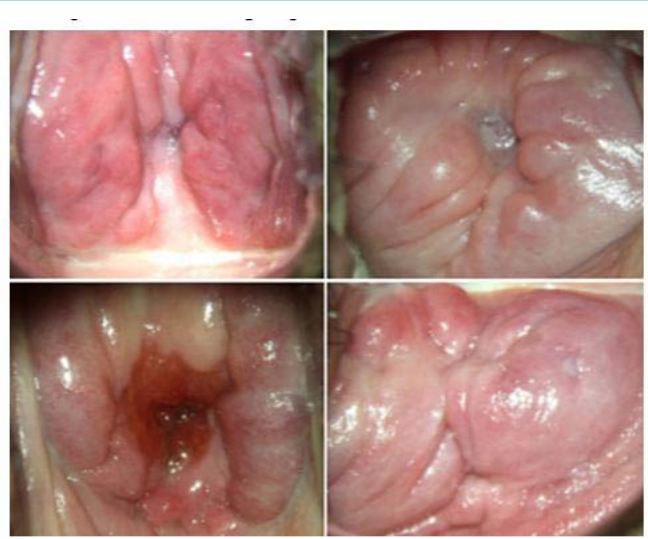

Figure 2: Colposcopy image of the CKC group.

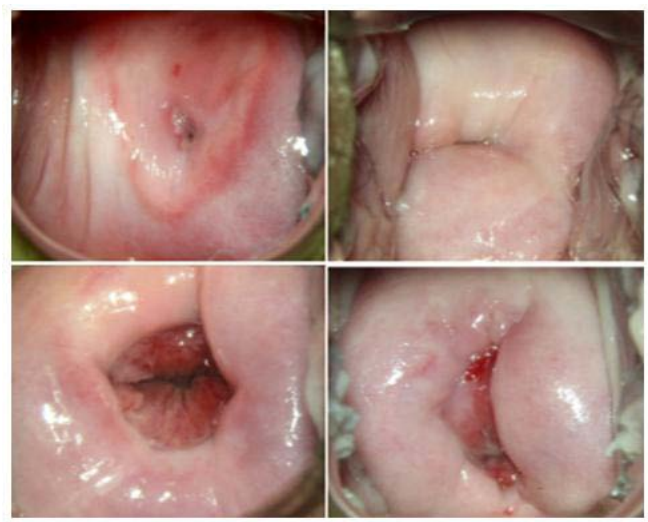

Figure 3: Colposcopy image of the HCKC group.

significant. Were not significantly different between the HCKC group (95\% and 0\% respectively) and the CKC group $(91.09 \%$ and $0.99 \%$ respectively) $(\mathrm{P}>0.05)$.

Postoperative infection, postoperative hemorrhage and the cervical adhesion rate were compared between the two groups (Table 4). Data show no significantly differences caused by HCKC or CKC treatment. All patients with postoperative infection were controlled by antibiotics. Postoperative hemorrhage was treated with vaginal packing.

Figure 2 and Figure 3 show the cervical of the HCKC group were closer to normal shape than the CKC group.

\section{Discussion}

$\mathrm{CKC}$ is one operative mode of conization. It removes cervical lesions with cone-shape [1]. It has been used for more than 200 years since Lisfranc did firstly cold-knife conization in 1815 [5]. CKC was not affecting pathologic diagnosis because it can get complete specimen [2]. Moreover, CKC have the advantage of high cure rate and low recurrence rate [3].

Despite CKC is a minor surgical procedure, it is annoying by suture difficulty due to the small surgical room and intraoperative bleeding, and difficulty in recovering to normal cervical shape [6]. Cold-knife conization pulse electrocautery can stanch bleeding quickly and 
shorten operation time [7], but imprecise electrocoagulation caused massive tissue be coagulated. Furthermore, a large number of necrotic tissues after electrocoagulation increased the chance of infection [8]. Moreover, cervical canal is still hard to hemostasis [9]. Transcervical Resection of Cervical Lesion (TCRC) is a newly used method to treat cervical lesions with few intraoperative bleeding. However, it requires operating skill and gets the fragmentary tissue specimens instead of the whole tissue which could affect pathologic diagnosis [10].

HCKC is an improved method for treating cervical intraepithelial neoplasia. It not only can get accurate pathological diagnosis because of complete tissue samples, but also has few intraoperative bleeding because of precise electrocoagulation hemostasis. Cervix recovery is much better and closer to normal cervical morphology [4].

Complications such as postoperative hemorrhage, infection and cervical adhesion are sometimes seen in patients after operation. Postoperative hemorrhage, the most common complication, was reported to be $10 \%$ [11]. Our study showed $4.65 \%$ and $1.80 \%$ in HCKC group and CKC group respectively, with no statistical difference $(\mathrm{P}>0.05)$. Cervical adhesion, which was reported to be $1 \%-5 \%$ in CKC treatment [12], in our study, this complication rate after CKC treatment was within these limits. However, no cervical adhesion happened in HCKC group.

\section{Conclusion}

In conclusion, HCKC is effective to treat patients with CINIII with the advantages of easily performing, time-conserving, less intraoperative bleeding, less expense, more HPF clearance, less cervical adhesion and beauty of shape recovery.

\section{References}

1. Qin yan, Xiaopin Wan, et al. Indications for conization of cervix. J Pract Obstet Gynecol. 2009; 25: 385-386.
2. Mathevet $P$, Chemali $E$, Roy $M$, et al. Long-term outcome of a randomized study comparing three techniques of conization: cold knife, laser, and LEEP. European Journal of Obstetrics \& Gynecology. 2003; 106: 214-218.

3. Reich O, Pickel H, Lahousen $\mathrm{M}$, et al. Cervical intraepithelial neoplasia III long-term outcome after cold-knife conization with clear margins. Obstetrics \& Gynecology. 2001; 97: 428-430.

4. Baojun Yang, Xuejun Zhang, Liming Feng, et al. Application of hysteroscopy assisted cold knife conization in the diagnosis and treatment of cervical intraepithelial neoplasia. Chinese Journal of endoscopy. 2007; 13: 811-814.

5. Siddall-Allum J. The History of Obstetrics and Gynaecology. Isis. 1995; 89: 533.

6. Hongying Dai, Yuying Duan, Zhongxin Wang, et al. Clinical experience of cold knife conization in the diagnosis and treatment of cervical intraepithelial neoplasia. Chinese Journal of cancer control. 2009; 16: 872-874.

7. Xianyou Yi, Yongzhong $\mathrm{Xu}$. Clinical comparative study of hemostasis by suture hemostasis and electrocoagulation for cervical cold knife conization. Chinese modern doctor. 2011; 49: 124-125.

8. Huixian Wang. Clinical analysis of 52 cases of bleeding after LEEP. Chinese community doctor. 2009; 11: 74 .

9. Tiecheng Lin, Gang Wang, Yubin Han, et al. A clinical study of loop electrosurgical excision procedure for chronic cervicitis. Chinese Journal of endoscopy. 2005; 11: 865-867.

10. Jiangang Shuai, Julang Dong, Ying Yan. Clinical observation of hysteroscopic cervical conization in the treatment of cervical intraepithelial neoplasia. Chinese Journal of reproductive health. 2010; 21: 141-143.

11. Kamat AA, Kramer P, Soisson AP. Superiority of electrocautery over the suture method for achieving cervical cone bed hemostasis. Obstetrics \& Gynecology. 2003; 102: 726-730.

12. Shuli Yang Wei Duan, Jinwei Miao. Comparison of two suture methods in cold knife conization of cervix. Journal of Chinese Physician. 2013; 41: 51-53. 